\title{
The Different Impact of VEGF-A and VEGF-D on Peritumoral Brain Edema
}

\author{
Zygmunt Siedlecki ${ }^{1 *}$, Danuta Rość ${ }^{2}$ and Maciej Śniegocki ${ }^{1}$ \\ ${ }^{1}$ Department of Neurosurgery and Neurotraumatology, Collegium Medicum in Bydgosz of Nicolaus Copernicus University in \\ Toruń, Poland
}

${ }^{2}$ Department of Pathophysiology, Collegium Medicum in Bydgosz of Nicolaus Copernicus University in Toruń, Poland

*Corresponding author: Zygmunt Siedlecki, Department of Neurosurgery and Neurotraumatology, University Hospital, Sklodowskiej-Curie 9 Street, 85-094 Bydgoszcz, Poland

ARTICLE INFO

Received: 慧 June 12, 2020

Published: 幽 June 24, 2020

Citation: Zygmunt S, Danuta R, Maciej S. The Different Impact of VEGF-A and VEGF-D on Peritumoral Brain Edema. Biomed J Sci \& Tech Res 28(3)-2020. BJSTR. MS.ID.004656

Abbreviations: BBB: Blood Brain Barrier; eNOS: Endo Thelialni Tricoxidesynthase; KS: Karnofsky Scale ; PIGF: Placenta Growth Factor; SAH: Subar Achnoid Hemorrhage; VEGF: Vascular Endothelial Growth Factor; VEGFR : Vascular Endothelial Growth Factor Receptor; VPF: Vascular Permeability Factor
ABSTRACT

The study consisted of VEGF-A and VEGF-D levels analysis in the blood samples in patients with brain tumors. VEGF is a family of angiogenic factors. This study evaluated the association between VEGF-A and VEGF-D levels and the extent of peritumoral brain edema. The study group was divided into patients with moderate and severe edema based on the Stenih off scale and its modification for posterior foss a tumors. It was found that plasma VEGF-A level in patients with severe brain edema was significantly higher $(\mathrm{Me}=25.42 \mathrm{pg} / \mathrm{ml})$ than in patients with moderate brain edema $(\mathrm{Me}=29.18$ $\mathrm{pg} / \mathrm{ml}$ ) - U Mann-Whitney test, $\mathrm{p}=0.0258$. It was also found that VEGF-D plasma level in patients with severe brain edema was significantly lower $(\mathrm{Me}=95.25 \mathrm{pg} / \mathrm{ml})$ than in patients with moderate brain edema $(\mathrm{Me}=183.57 \mathrm{pg} / \mathrm{ml})$ - Mann-Whitney test, $p=0.0034$. It was also found that the plasma concentration of VEGF-D in patients with better functional status was significantly higher $(\mathrm{Me}=185.47 \mathrm{pg} / \mathrm{ml})$ than the concentration in patients with worse functional status $(\mathrm{Me}=111.34 \mathrm{pg} / \mathrm{ml})-\mathrm{U}$ Mann-Whitney test, $\mathrm{p}=0.0428$. The above results proved that VEGF-A and VEGF-D present omni directional association with peritumoral brain edema in patients with intracranial tumors. VEGF-A promotes the formation of peritumoral edema, while the relationship between VEGF-D and brain edema seems to be the opposite of VEGF-A.

Keywords: Brain Tumor; Brain Edema; VEGF

\section{Introduction}

Vascular endothelial growth factor (VEGF) plays important role in tumor development and patophysiology [1]. It is main angiogenic factor and promotes new blood vessels formation and also causes the permeability of blood vessels and inconsequence edema $[1,2]$. In the literature it is also referred to as vascular permeability factor (VPF). Often in references it is named VEGF / VPF [1]. VEGF interacts via VEGFR1, VEGFR2, and VEGF3 receptors. These are receptors with tyrosine kinase activity. VEGF belongs to the platelet-derived growth factors family [2]. The most important form is VEGF-A and has main biological activity in angiogenesis and vascular permeability [1,2]. The importance of VEGF-A in promoting brain edema in tumors has been reported by many authors [3]. VEGF-A causes blood-brain barrier (BBB) permeability and passage of fluid from blood vessels to brain tissue [3]. The main receptor responsible for VEGR-A activity is VEGFR2. The VEGF family also includes VEGF-B, VEGF-C, VEGF-D and Placenta Growth Factor (PIGF), other forms such as VEGF-E and VEGF-D in animal models and their role is not known [4]. In past decades, attention has been gained to the biological significance of VEGF-D as an equally important biologically important factor [5]. VEGF-D effects on the cell via the VEGFR3 receptor. VEGFR3 does not react with VEGF-A and this may indicate the different biological activity of VEGF-D and VEGF-A [4,5]. The exact biological significance of VEGF-D is still under investigation by many authors [4]. VEGF-A and VEGF-D are significantly described in the literature for the progression of colorectal cancer [6]. The aim of this study was to analyze the association of VEGF-A and VEGF-D in brain edema in patients with intracranial tumors, based on the plasma level analysis of these factors. 


\section{Material and Methods}

In order to conduct the research, the approval of the Bioethics Committee of Nicolaus Copernicus University Collegium Medicum in Bydgoszcz was obtained, its number being: KB-665/2009.The study included 47 adult patients $(\mathrm{Mb}=57.52)$ of bothsexes (22 women, 25 men) treated surgically for intra cranial tumors in the Department of Neurosurgery and Neurotraumatology in Collegium Medicum in Bydgoszcz of Nicolaus Copernicus University in Toruń. The diagnosis of intracranial tumors was based on medical history, physical examination, neuroimaging. Although patients with solitary intracranial metastatic tumors were in the study group, the study excluded patients with disseminated metastatic disease with multiple metastases and patients who under went surgery within less than 30 days prior to admission to the Neurosurgery and Neurotraumatology Department. Also excluded were patients with pre-existing consciousness disorders which prevented them from giving informed consent. In study group $78.72 \%$ tumors were located supratentorial and $14.89 \%$ tumors in the posterior cranialfossa. In $6.38 \%$ cases the tumors were located in sellar and parasellar region.The size of peritumoral brainedema was evaluated in all 47 patients. Using the Steinhoff scale, brain edema was evaluated in the all cases of supratentorial, sellar and parasellar tumors. Steinhoff scaleevaluates the size of brain edema around brain tumors. It is four-grade scale.In this manuscript for patients with posterior fossa tumors the modification of Steinhoff scale elaborated by authors was used. Authors referred the Steinhoff scale to posterior tumors to accurately reflect the extent of edema and its clinical implications. Steinhoff scale and its own modification for posterior fossa tumors is presented in (Table 1).

Table 1: Brain edema in Steinhoff scale and its own modification for posterior fossa tumors.

\begin{tabular}{|c|c|c|}
\hline Degree of Brainedema & Steinh off Scale & Modification for Posterior Fossa Tumors \\
\hline I & $\leq 2.5 \mathrm{~cm}$, envelope shape & $\leq 1 \mathrm{~cm}$, envelope shape \\
\hline II & It does not exceed half the brain hemisphere & It does not exceed half the cerebellar hemisphere \\
\hline III & It exceeds half the brain hemisphere & Edema covering significance region of posterior fossa \\
\hline IV & $\begin{array}{c}\text { Edema in the contralateral hemisphere of the } \\
\text { brain }\end{array}$ & \begin{tabular}{c} 
It excerebellar hemisphere \\
\hline
\end{tabular}
\end{tabular}

Based on the above scale, the study group was divided into two subgroups: 1 . patients with moderate brain edema (1. and 2. in Steinhoff scale and its modification), 2. Patients with severe brain edema (3. and 4. in Steinhoff scale and its modification). Sub group 1. Consisted of 32 patients (68.08\%) and subgroup 2. consisted of 15 patients $(31.91 \%)$. The clinical and functional status of patients was also assessed on the basis of the Karnofsky Scale (KS; 0-100). The examined patients were divided into two groups: 1 . patients in better functional condition (KS $=90-100$ ) consisted of 29 patients $(61.70 \%)$ and 2 . patients in worse functional condition (KS $=\leq 80)$ consisted of 18 patients (38.30\%). In the study blood samples were taken within 24 hours to 1 hour before the operation. Venous blood was collected from direct punctures of the basilicvein, median cubitalveinor median antebrachialvein, following skin disinfection with the antibacterial agent Kodan ${ }^{\circledR}$ Tinktur Forte, using the Vacuette ${ }^{\circledR}$ vacuum blood collection system from Greiner Bio-One. Venous blood was collectedintotwo plastic tubes with sodiumcitrateat a concentration of $3.2 \%$ edetic acid (EDTA) in a ratio of 1:10- one part of anticoagulant to nineparts of blood in the tube. The samples were collected in accordance with the current blood collection procedure used at the Department of Neurosurgery and Neurotraumatology of Nicolaus Copernicus University.

In order to obtain platelet-poor plasma, the blood samples were centrifuged in a refrigerated centrifuge at $4{ }^{\circ} \mathrm{C}$ for 20 minutesat the speed of $3000 \mathrm{rpm}$. The obtained citrate plasma and EDTA plasma was separated into portions of approximately $200 \mu \mathrm{l}$ in Eppendorftubes. The material was frozenat $-80^{\circ} \mathrm{C}$ until the time of testing. Concentrations of individual factors were determined using reagent kits from R\&D and Bender Med Systems. In the plasma, the level of VEGF-A was marked using Human VEGF Bender Med Systems reagent with no reference values provided by the manufacturer for VEGF-A. The level of VEGF-D was marked using R\&D Human VEGF-D reagent; the reference values were $0-437 \mathrm{pg} / \mathrm{dl}$. The study used the ELISA immunoassay method. The tests were carried out in the Hemostatic Disorders Laboratory of the Department of Pathophysiology, Nicolaus Copernicus University's Medical School in Bydgoszcz. For determining the parameters under investigation, in all cases, the study only used samples of patient blood collected in order to carry out laboratory tests required for diagnostics and therapy during hospitalization. All patients participating in the study had been informed of the nature and purpose of the study and gave informed consent for further measurements of plasma VEGF-A and VEGF-D levels.

\section{Statistical Analysis}

The statistical analysis was carried out using Stat Soft $₫$ statistical program STATISTICA 8.0. The distribution of the analyzed parameters was different from the normal distribution, therefore nonparametric statistics were used: Kruskal-Wallis and Friedman test. The significance of differences between two groups was assessed using the Mann-Whitney $U$ test for independent variables and Wilcoxon for dependent variables. The significance level of $\mathrm{p}$ $<0.05$ was assumed to be statistically significant. The Spearman correlation coefficients used in the correlation analysis were also considered statistically significant for $\mathrm{p}<0.05$. The interdependence of the analyzed parameters is presented graphically in the form of scatterplots with linear regression curves. 


\section{Results}

It was found that plasma VEGF-A level in patients with severe brain edema was significantly higher $(\mathrm{Me}=25.42 \mathrm{pg} / \mathrm{ml})$ than in patients with moderate brain edema $(\mathrm{Me}=29.18 \mathrm{pg} / \mathrm{ml})-\mathrm{U}$ Mann-Whitney test, $\mathrm{p}=0.0258$. It was also found that VEGF-D plasma level in patients with severe brain edema was significantly lower $(\mathrm{Me}=95.25 \mathrm{pg} / \mathrm{ml}$ ) than in patients with moderate brain edema $(\mathrm{Me}=183.57 \mathrm{pg} / \mathrm{ml})-$ Mann-Whitney test, $\mathrm{p}=0.0034$.
The results of VEGF-A and VEGF-D plasma levels associated with brain edema are both presented in (Table 2). (Figure 1) presents VEGF-A plasma level associated with brain edema and (Figure 2). Presents this association regarding VEGF-D. It was also found that the plasma concentration of VEGF-D in patients with better functional status was significantly higher $(\mathrm{Me}=185.47 \mathrm{pg} / \mathrm{ml}$ ) than the concentration in patients with worse functional status (Me $=111.34 \mathrm{pg} / \mathrm{ml}$ ) $-\mathrm{U}$ Mann-Whitney test, $\mathrm{p}=0.0428$. These results are presented in (Table 3) and (Figure 3).

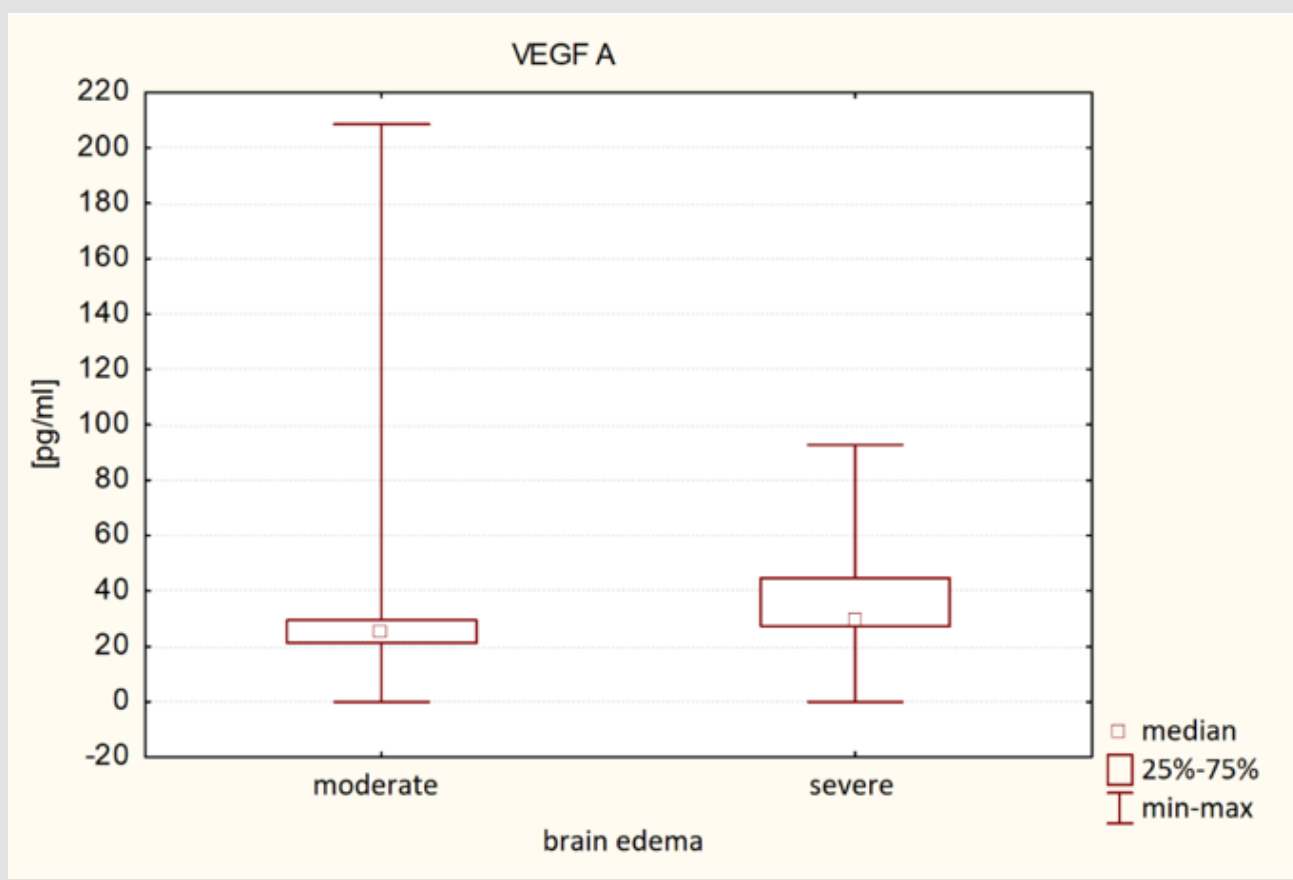

Figure 1: VEGF-A associated with brain edema.

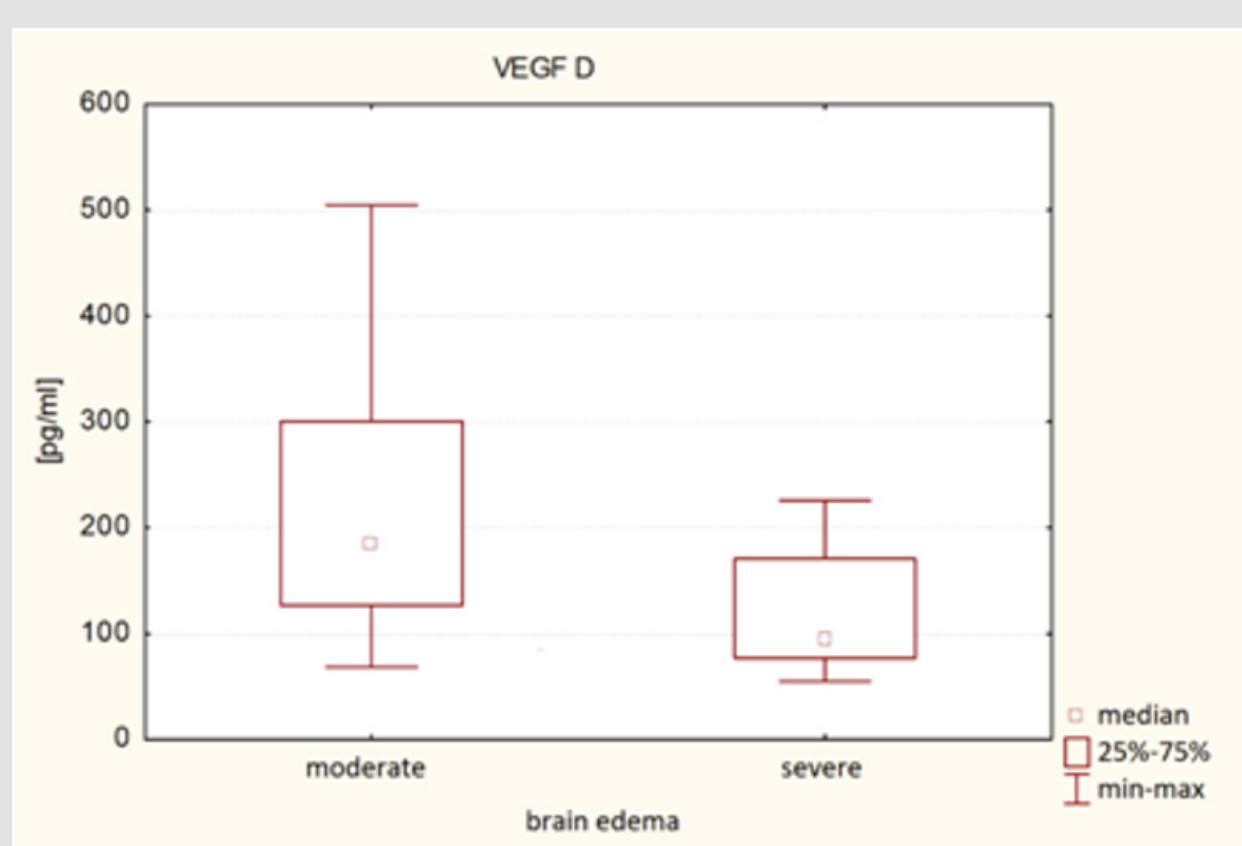

Figure 2: VEGF-D associated with brain edema. 


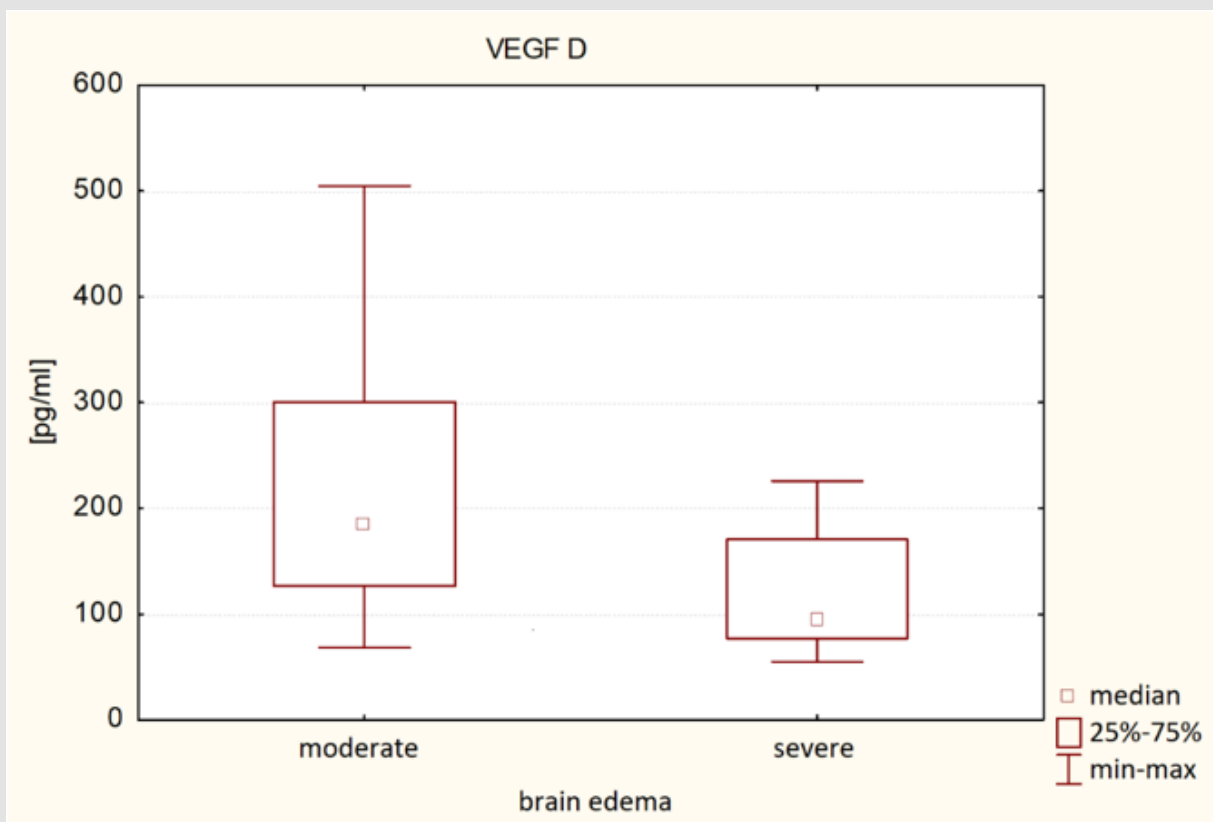

Figure 3: VEGF-D associated with functional status of patients.

Table 2: VEGF-A and VEGF-D associated with peritumoral brain edema extent.

\begin{tabular}{|c|c|c|c|c|}
\hline \multirow{2}{*}{ Measured Parameter } & Descriptive Statistics & \multicolumn{2}{|c|}{ Brain edema } & p \\
\cline { 2 - 4 } & & Moderate & Severe & \\
\hline \multirow{3}{*}{$\begin{array}{c}\text { VEGF-A } \\
{[\mathrm{pg} / \mathrm{ml}]}\end{array}$} & $\mathrm{N}$ & 32 & 27,38 & $0,0258^{*}$ \\
\cline { 2 - 5 } & $\mathrm{Q} 1$ & 21,20 & 29,18 & \\
\cline { 2 - 5 } & $\mathrm{Me}$ & 25,42 & 44,70 & \\
\hline \multirow{3}{*}{$\begin{array}{c}\text { VEGF-D } \\
{[\mathrm{pg} / \mathrm{ml}]}\end{array}$} & $\mathrm{Q}$ & 29,57 & 15 & $0,0034^{*}$ \\
\cline { 2 - 5 } & $\mathrm{Q} 1$ & 126,55 & 77,19 & \\
& $\mathrm{Me}$ & 183,57 & 95,25 & \\
\end{tabular}

Table 3: VEGF-D associated with functional status of patients.

\begin{tabular}{|c|c|c|c|c|}
\hline \multirow{2}{*}{ Measured Parameter } & \multirow{2}{*}{ Descriptive Statistics } & \multicolumn{2}{|c|}{ Karnofsky Scale } & \multirow{2}{*}{ p } \\
\cline { 3 - 5 } & & $\mathbf{9 0 - 1 0 0}$ & $\mathbf{3}$ & \\
\hline VEGF-D & $\mathrm{N}$ & 29 & 78,67 & $0,0428^{*}$ \\
\hline$[\mathrm{pg} / \mathrm{ml}]$ & $\mathrm{Q} 1$ & 130,48 & 111,34 & \\
\hline & $\mathrm{Me}$ & 185,47 & 183,57 & \\
\hline
\end{tabular}

\section{Discussion}

The effect of VEGF on brain edema formation is described in many references in the literature. VEFG-A, as the most common form of VEGF, has the best known mechanism of impact on brain edema and its clinical implications [1-6]. Gerstner el. [7] emphasizes that the most brain tumors over secrete VEGF-A [7]. Elevated level of VEGF-Aleads to anabnormally permeable tumor vasculature and allows fluid to leak from the intravascular space into the brain tissue [7]. It causes vaso geniccerebraledema and increased interstitial fluid pressure. According to Gerstner et al. Cerebraled emacause significant morbidity and mortality and isassociated with neurological condition [7]. Gerstner also emphasizes that VEGF-A is potential target in brain edema treatment because agents that block the VEGF-A path way are able to decrease vascular permeability by restoring the abnormal tumor vasculature to a morenormal state [7]. It coincides with the results of our study, where high levels of VEGF-A were associated with severe brain edema around the tumor. Wang et al. [8] based rat model emphasizes that VEGFis the major regulator of micro vascular permeability [8]. Acoording to Wang et al. BBB permeability was significantly increased following 
treatment with VEGF. It has also been well documented that VEGF expression is increased in various type of cells, including neurons, endo the lial cells, astrocytes, pial cells in rats [8].

VEGF-induced permeability depends on the VEGFR2-mediated endo the lialnitricoxidesynthase (eNOS) pathway by down regulating the expression of tight junction proteins [1-4,8]. In addition, the tight junction between endothelial cells is the key element in BBB permeability, and disruption of tight junctions leads to BBB breakdown on rat model [8]. Liu et al. [9] describes that VEGF has been implicated in the pathogenesis of brain edema formation after experimental subarachnoid hemorrhage (SAH) [9]. His study suggests that VEGF is involved in brain edema formation after SAH, and that anti-VEGF antibody an decrease BBB permeability, suppress brain edema formation, and improve functional outcome after $24 \mathrm{~h}$ of SAH [9]. In our study no association was found between VEGF-A and the patients clinical state of patients, also our study VEGF impact on peritumoral brain edema in cancer patients was described while Liu studied VEGF-A in brain edema after SAH. However, regarding association between VEGF and brain edema, our results are the same as Liu et al. [9] Carlson et al. [10], similarly to our study, examined VEGF-A in brain edema in tumor cases. Similarly to other authors Gerstner et al. [7-9] and like our study Carlson proved that VEGF-Ais a potent mediator of vascular permeability [10]. According to Carlson et al. VEGF inhibition reduces edema and tumor burden in some patients with malignantglioma, where as others show no response. The role of VEGF expression in edema production and the relationship to survivalis not well understood [10].

Carlson postulated that there are both VEGF-dependent and VEGF-independent pathways of edema production in gliomas and may explain whye demais not reduced in some patients following anti-VEGF treatment [10]. Although most references refer to the role of VEGF-A in brain edema and the literature data is consistent that VEGF increases brain edema by increasing vascular permeability [6-10], there are also reports on the role of VEGF-D in brain edema and vascular permeability. Our study showed that higher VEGF-D plasma levels were associated with less brain edema and better functional status. Schluter et al. [11] showed on mice model that in vivo VEGF-D supplementation via preserved the structural and functional integrity of retinal ganglion cells agains texcito toxicity and, additionally spared endothelial cells from degeneration [11]. Viral-mediated suppression of expression of the VEGF-D-binding receptor VEGFR3 in retinal ganglion cells revealed that VEGF-D exerts its protective capacity directly on retinal ganglion cells, while protection of endothelial cells is the result of upheld neuronalintegrity [11]. These findings suggest that VEGF-D supplementation might be a novel, clinically applicable approach for neuronal and vascular protection [11]. However, the study by Schluter et al. it was on mice model, the conclusions from it coincide with our results, which indicate that a higher concentration of VEG-D promotes less brain edema. Study of Schluter et al. may potentially explain that such effect is due to increased protection and vascular integrity [11], moreover, Schluter shows that VEGF-D has a positive effect on neuronal integrity [11].

In our work, it has been shown that a higher concentration of VEGF-D is associated with a better clinical and functional state. Von Moos [12] obtained different results in the subject of VEGF-D in vessel permeability. In her work, she examined VEGF-D levels in fluid overloaded patients treated by hemo dialysis. Fluid over load was defined by bio impedance analysis [12]. He study proved that fluid overload correlated positively with VEGF-D levels [12]. Von Moos also showed that VEGF-D is a potential over hydration and prognostic marker. People with high VEGF-D levels were in worse clinical condition and the prognosis was worse [12]. The results of von Moos are different from the results of our work.

\section{Conclusion}

VEGF-A and VEGF-D present omni directional association with peritumoral brain edema in patients with intracranial tumors. VEGF-A promotes the formation of cerebral edema, which is consistent with literature data. VEGF-A promotes blood vessels permeability. While the relationship between VEGF-D and brain edema seems to be the opposite of VEGF-A. Patients with elevated plasma VEGF-D levels had less brain edema and were in better clinical state. Data about VEGF-D impact on vascular permeability are divergent, although there are reports that VEGF-D protects vascular integrity, which is consistent with the results of this manuscript. To understand the VEGF-D impact on brain edema and vascular and cellular integrity, further research at the molecular level beyond this manuscript must be performed.

\section{Declarations}

\section{Ethics Approval and Consent to Participate}

The experiment was approved by the Bioethics Committee of the Ludwik Rydygier Collegium Medicum in Bydgoszcz (KB$665 / 2009$ ). Written inform edconsent was obtained from the patientsfor publication of thismasuscript and anyaccompanying data. A copy of the writtenconsentisavailable for review by the Editor of thisjournal.

\section{Consent for publication}

Written in form edconsent was obtained from the all participians for publication of this masuscript and any accompanying data.

\section{Availability of Data and Materials}

All relevant data are within the paper.

\section{Competing Interests}

The authors declare that they have no conflict of interest. 


\section{Funding}

The study was financed from own funds of the Neurosurgery of Collegium Medicum in Bydgoszcz. The authors received no specific founding for this work.

\section{Authors' contributions:}

1. Protocol/project development: Siedlecki

2. Data collection and management: Siedlecki

3. Data analysis: Siedlecki

4. Manuscript writing/editing: Siedlecki

\section{Acknowledgement}

The authors thank the technicians and laboratory staff of the Department of Pathophysiology for the huge contribution of work and professionalism in HGF determination in blood samples.

\section{Conflict of Interest}

The authors declare no conflict of interest.

\section{References}

1. Yamada Y, Nezu JI, Shimane M (1997) Molecularcloning of a novel vascular endothelial growth factor, VEGF-D. Genomics 42(3): 483-488.

2. Neufeld G, Cohen T, Gengrinovitch S (1999) Vascular endothelial growth factor (VEGF) and its receptors. The FASEB journal 13(1): 9-22.

3. Berhouma M, Jacquesson T, Jouanneau E (2019) Pathogenesis of peritumoraledema in intracranial meningiomas. Neurosurgical review 42(1): 59-71.

ISSN: $2574-1241$

DOI: $10.26717 /$ BJSTR.2020.28.004656

Zygmunt Siedlecki. Biomed J Sci \& Tech Res

(C) This work is licensed under Creative Commons Attribution 4.0 License

Submission Link: https://biomedres.us/submit-manuscript.php
4. Achen MG, Jeltsch M, Kukk E, T Mäkinen, A Vitali, et al. (1998) Vascular endothelial growth factor D (VEGF-D) is a ligand for the tyrosinekinases VEGF receptor 2 (Flk1) and VEGF receptor 3 (Flt4). Proceedings of the National Academy of Sciences 95(2): 548-553.

5. Li X, Eriksson U (2001) Novel vegf family members: Vegf-b, vegf-c and vegf-d. The international journal of biochemistry \& cell biology 33(4): 421-426.

6. George ML, Tutton MG, Janssen F, A Arnaout, A M Abulafi, et al. (2001) Vegf-a, vegf-c, and vegf-d in colorectal cancer progression. Neoplasia (New York, NY) 3(5): 420

7. Gerstner ER, Duda DG, Di Tomaso E, Peter A Ryg, Jay S Loeffler, et al. (2009) VEGF inhibitors in the treatment of cerebraledema in patients with brain cancer. Nature reviews Clinical oncology 6(4): 229-236.

8. Wang Q Deng Y, Huang L, Wenxin Zeng, Shenglong Chen, et al. (2019) Hypertonic saline down regulates endothelial cell-derived VEGF expression and reduces blood-brain barrier permeability induced by cerebralis chaemia via the VEGFR2/eNOS pathway. International journal of molecular medicine 44(3): 1078-1090.

9. Liu L, Fujimoto M, Kawakita F, Naoki Ichikawa, Hidenori Suzuki, et al. (2016) Vascular endothelial growth factor in brain edema formation after subarachnoid hemorrhage. In Brain Edema XVI (pp. 173-177).

10. Carlson MR, Pope WB, Horvath S (2007) Relationship between survival and edema in malignant gliomas: Role of vascular endothelial growth factor and neuronal pentraxin 2. Clinical Cancer Research 13(9): 25922598.

11. Schlüter A, Aksan B, Diem R, Richard Fairless, Daniela Mauceri, et al. (2020) VEGFD Protects Retinal Ganglion Cells and, consequently, Capillaries against Excitotoxic Injury. Molecular Therapy-Methods \& Clinical Development 17: 281-299.

12. von Moos S, Segerer S, Davenport A (2020) Vascular endothelial growth factor D is a biomarker of fluid overload in haemodialysispatients. Nephrology DialysisTransplantation.

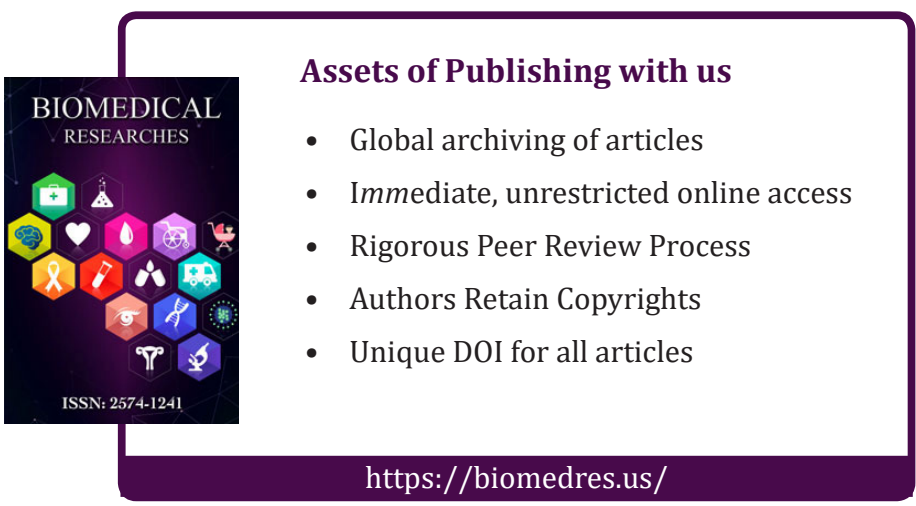

